\title{
Modelagem matemática de secagem de frutos de Physalis
}

\author{
P. G. SILVEIRA*, J. R. J. JUNQUEIRA, \\ OLIVEIRA.H. M, MELLO JR. R.E e J. L.G. CORRÊA \\ Universidade Federal de Lavras, Departamento de Ciência dos Alimentos \\ E-mail para contato*: pagiarolla@gmail.com
}

\begin{abstract}
RESUMO - A secagem convectiva de frutos de physalis (Physalis peruviana) foi realizada à temperatura de $60^{\circ} \mathrm{C}$ e velocidade de ar de $2 \mathrm{~m} . \mathrm{s}^{-1}$ sem e com diferentes pré-tratamentos (congelamento rápido com nitrogênio líquido, congelamento lento). A secagem foi realizada até o teor de umidade final de 0,08 $\pm 0,02 \mathrm{~kg} \cdot \mathrm{kg}^{-1}$ (b.s). Aos dados de cinética de secagem foram aplicadas seis Equações empíricas (Page, Page Modificado, Logarítmica, Midilli, Wang \& Singh e Parabólica). Dentre as Equações avaliadas, a Equação de Wang \& Singh foi a que melhor se ajustou aos dados experimentais, com valores de $\mathrm{R}^{2}$ maiores que 0,99 .
\end{abstract}

\section{INTRODUÇÃO :}

O physalis (Physalis peruviana) é um fruto comestível da família das solanáceas rico em vitaminas A e C, fósforo e ferro, além de apresentar flavonoides, alcaloides e fitoesteróides. Comumente consumida na forma in natura, o fruto apresenta sabor agridoce. É também ingrediente para molhos, compotas, doces, geleias, sorvetes e licores. Folhas, frutos e raízes são usados na medicina popular para combater diabetes, reumatismo crônico, doenças de pele, da bexiga e do fígado (Ramadan, 2011).

A superfície do fruto é coberta por uma mistura de substâncias que a tornam impermeável (álcoois, alcanos, aldeídos, cetonas e ésteres feitos de ácidos graxos de cadeia longa) e dificultam a remoção de umidade do interior do fruto. Dentre os processos de conservação de alimentos, a secagem é um dos mais importantes contribuindo para extensão da vida útil do produto, distribuição em todas as regiões e épocas do ano e diferenciação de produtos (Silva e Corrêa, 2005; Corrêa et al., 2011). Devido à presença desses compostos na camada externa, tratamentos na casca são necessários para facilitar a retirada de umidade do fruto (Doymaz e Özdemir, 2014). Durante o congelamento rápido ocorre a formação de muitos cristais pequenos promovendo uma estrutura mais aberta quando comparada ao congelamento lento (Dermesonlouoglou et al., 2008) sendo também verificado em um trabalho complementar sobre secagem de physalis com o uso do congelamento como prétratamento (Junqueira et al., 2017).

A modelagem matemática durante a secagem de alimentos é aplicada na avaliação do comportamento da secagem. Dentre as equações empregadas para a modelagem dos dados experimentais de secagem, semi-teóricas apresentam maior compromisso com os fenômenos envolvidos durante a transferência de calor e de massa, são facilmente aplicáveis. A maioria destas equações são derivadas da solução analítica da segunda lei de Fick (Cranck, 1975). 
O objetivo deste trabalho foi ajustar seis equações empíricas (Page, Page Modificado, Logarítmica, Midilli, Wang \& Singh e Parabólica) aos dados experimentais de secagem de frutos de physalis pré-tratados fisicamente por congelamento em diferentes taxas (congelamento rápido com nitrogênio líquido e congelamento lento em freezer doméstico) e sem pré-tratamento (Ketata et al., 2013; Zielinska e Michalska, 2016).

\section{Material e métodos}

Os physalis foram coletados na Universidade Federal de Lavras, Lavras, Minas Gerais, Brasil. Os frutos foram escolhidos visualmente por tamanho uniforme, peso, cor, intensidade e firmeza. Antes de cada pré-tratamento, o cálice dos frutos foi manualmente retirado. $\mathrm{O}$ teor inicial de umidade $\left(3,11 \pm 0,01 \mathrm{~kg} \cdot \mathrm{kg}^{-1}\right.$ em base seca (b.s.) foi determinado em estufa à vácuo a $70^{\circ} \mathrm{C}$ (AOAC, 2010).

Os pré-tratamentos na superficie do fruto foram: (i) congelamento rápido por imersão em nitrogênio líquido $\left(-196^{\circ} \mathrm{C}\right)$ durante $10 \mathrm{~s}$ seguido de descongelamento a $25^{\circ} \mathrm{C}$, (ii) congelamento lento, inserindo os frutos num congelador doméstico (modelo Electrolux, DC51, Brasil) a $-18 \pm 2{ }^{\circ} \mathrm{C}$, seguido de descongelamento a $25^{\circ} \mathrm{C}$ durante 2 horas (Ketata et al., 2013). Frutos tratados e não tratados foram submetids à secagem convectiva em secador de túnel (Eco Engenharia Educacional, modelo MD018, Brasil) com fluxo paralelo a $60^{\circ} \mathrm{C}$ e 2 $\mathrm{m} . \mathrm{s}^{-1}$. A massa inicial da amostra de frutos foi $0,100 \pm 0,003 \mathrm{~kg}$, o que corresponde entre $30 \mathrm{e}$ 35 frutos. Os experimentos foram realizados até o teor de umidade final de $0,08 \pm 0,02 \mathrm{~kg} \cdot \mathrm{kg}^{-1}$ (b.s). Durante a secagem, a massa das amostras foi monitorada empregando-se uma balança digital (Marte Científica, modelo AD33000, Brasil) acoplado ao suporte de amostras. Os experimentos foram efetuados em triplicata.

Para modelagem matemática, o teor de umidade adimensional (MR) foi calculado, conforme a Equação 1:

$$
M R=\frac{X_{t}-X_{e q}}{X_{0}-X_{e q}} \approx \frac{X_{t}}{X_{0}}
$$

Onde $X_{t}, X_{0}$ e $X_{\text {eq }}$ são os teores de umidade no tempo $(\mathrm{t})$, inicial e de equilíbrio [ $\mathrm{kg} \mathrm{kg}^{-1}$ (b.s)], respectivamente. Nas condições experimentais, o valor de $X_{\text {eq }}$ é relativamente pequeno em comparação a $\mathrm{X}_{0}$, portanto, seu valor foi considerado zero neste estudo (Doymaz, 2016). As seis equações empíricas (Junqueira et al., 2016) aplicadas na secagem de frutos de physalis estão apresentadas na Tabela 1.

Tabela 1- Equações empíricas de curva de secagem.

\begin{tabular}{llr}
\hline Modelo & Equação & Número \\
\hline Wang \& Singh & $\mathrm{MR}=1+\mathrm{at}+\mathrm{bt}^{2}$ & (2) \\
Parabólico & $\mathrm{MR}=\mathrm{a}+\mathrm{bt}+\mathrm{ct}^{2}$ & (3)
\end{tabular}


Page $\quad M R=\exp \left(-k t^{\mathrm{n}}\right)$

Page modificado $\quad \mathrm{MR}=\exp \left[-(\mathrm{kt})^{\mathrm{n}}\right]$

Midilli

$\mathrm{MR}=\mathrm{a} \cdot \exp \left(-\mathrm{kt}^{\mathrm{n}}\right)+\mathrm{bt}$

Logarítmico

$\mathrm{MR}=\mathrm{a} \cdot \exp \left(-\mathrm{kt}^{\mathrm{n}}\right)+\mathrm{b}$

Onde MR é o teor de umidade adimensional, a, b, c, n e k são coeficientes de ajuste e té

o tempo, em segundos.

As análises estatísticas dos dados experimentais foram realizadas utilizando o software Statistica (Statistica 8.0, Statsoft Inc., Tulsa, OK). O coeficiente de determinação $\left(\mathrm{R}^{2}\right)$, erro quadrático médio $(\mathrm{EQM})$ e qui-quadrado reduzido $\left(\chi^{2}\right)$ foram utilizados para determinar a qualidade do ajuste das Equações. Estes parâmetros podem ser calculados usando as Equações 8 e 9:

$$
\begin{aligned}
& E Q M=\sqrt{\left[\frac{1}{N} \sum_{i=1}^{N}\left(M R_{p r e d, i}-M R_{\text {exp }, i}\right)^{2}\right]} \\
& \chi^{2}=\sum_{i=1}^{N} \frac{\left(M R_{p r e d, i}-M R_{\text {exp }, i}\right)^{2}}{N-n^{\prime}}
\end{aligned}
$$

onde $\mathrm{MR}_{\exp }$ e $\mathrm{MR}_{\text {pred }}$ representam os teores de umidade adimensional experimentais e preditos, respectivamente; $\mathrm{N}$ é o número de observações e n ' é o número de constantes das Equações.

Como esses parâmetros apresentados não são suficientes para avaliar modelos matemáticos não-lineares, o módulo de desvio relativo médio (P\%) também foi usado para selecionar a melhor Equação:

$$
P(\%)=\frac{100}{n^{\prime}} \sum_{i=1}^{N}\left|\frac{M R_{\text {exp }}-M R_{p r e d}}{M R_{\exp }}\right|
$$

\section{Resultados e discussão:}

Os teores adimensionais de umidade obtidos nos diferentes tratamentos foram ajustadas a seis Equações empíricas listadas na Tabela 1. Os coeficientes das Equações de secagem e os critérios de comparação são apresentados na Tabela 2.

Tabela 2 - Coeficientes das Equações de secagem e critérios de comparação.

\begin{tabular}{cccccc}
\hline Equação & Tratamentos & Coeficientes & $\mathrm{R}^{2}$ & RMSE & $\chi^{2}$ \\
\hline \multirow{2}{*}{ Page } & $\begin{array}{c}\text { Sem } \\
\text { tratamento }\end{array}$ & $\begin{array}{c}\mathrm{k}=0,000017 \\
\mathrm{n}=1,140256\end{array}$ & 0,9918 & 0,0293 & 0,0009 \\
& & & & & \\
\hline
\end{tabular}




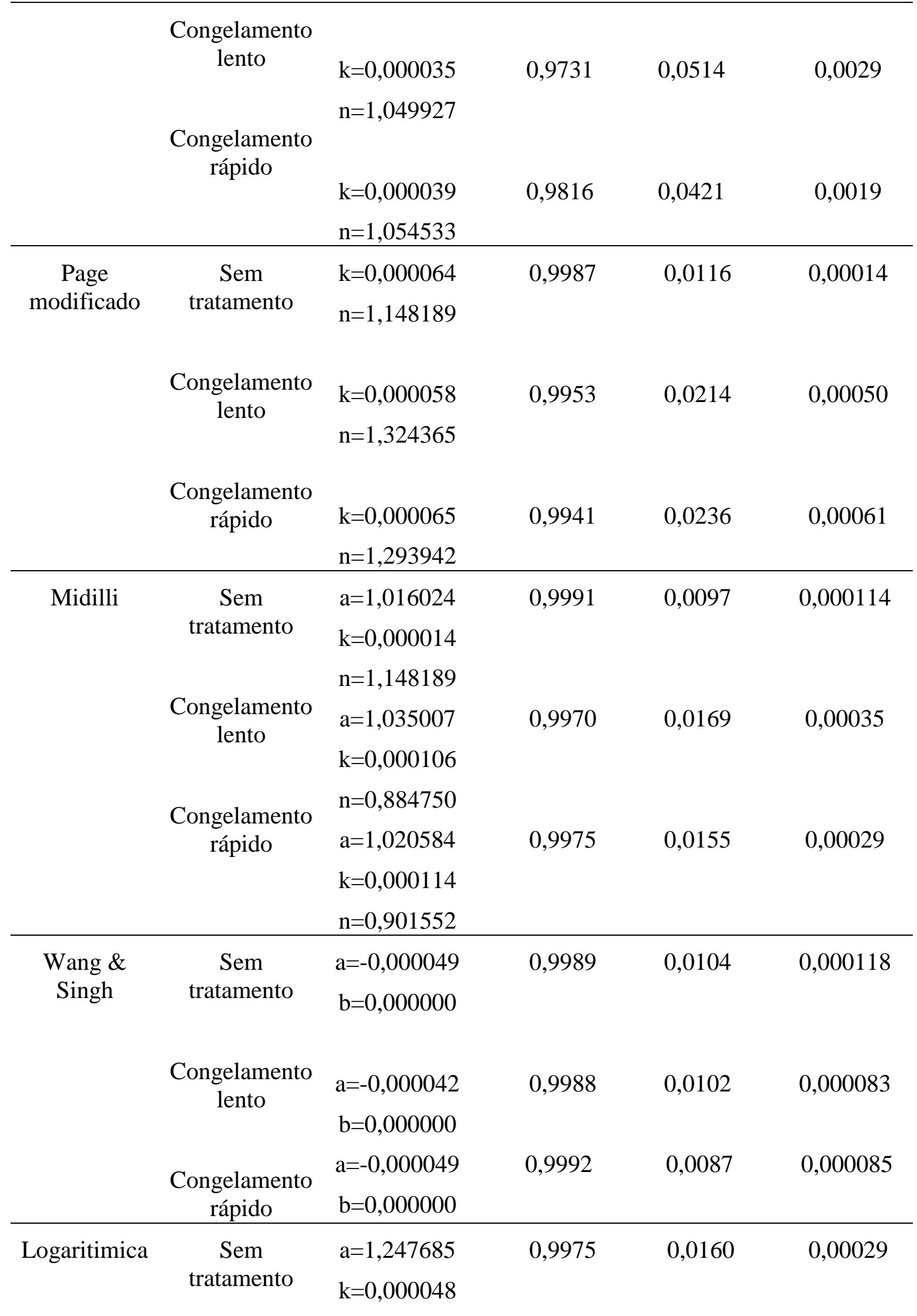




\begin{tabular}{|c|c|c|c|c|c|}
\hline & \multirow{3}{*}{$\begin{array}{c}\text { Congelamento } \\
\text { lento }\end{array}$} & $c=-0,210065$ & \multirow{3}{*}{0,9971} & \multirow{3}{*}{0,0166} & \multirow{3}{*}{0,00032} \\
\hline & & $\mathrm{a}=1,381580$ & & & \\
\hline & & $\mathrm{k}=0,000037$ & & & \\
\hline & \multirow{4}{*}{$\begin{array}{l}\text { Congelamento } \\
\text { rápido }\end{array}$} & $c=-0,342378$ & & & \\
\hline & & $a=1,318081$ & 0,9978 & 0,0144 & 0,00024 \\
\hline & & $\mathrm{k}=0,000044$ & & & \\
\hline & & $c=-0,298292$ & & & \\
\hline \multirow[t]{9}{*}{ Parabólico } & \multirow{3}{*}{$\begin{array}{c}\text { Sem } \\
\text { tratamento }\end{array}$} & $a=1,020969$ & 0,9996 & 0,0061 & 0,00004 \\
\hline & & $b=-0,00005$ & & & \\
\hline & & $c=0,000000$ & & & \\
\hline & \multirow{2}{*}{$\begin{array}{c}\text { Congelamento } \\
\text { lento }\end{array}$} & $a=1,016080$ & 0,9992 & 0,0084 & 0,000083 \\
\hline & & $b=-0,00004$ & & & \\
\hline & \multirow{4}{*}{$\begin{array}{l}\text { Congelamento } \\
\text { rápido }\end{array}$} & $\mathrm{c}=0,000000$ & & & \\
\hline & & $a=0,996795$ & 0,9992 & 0,0087 & 0,000087 \\
\hline & & $b=-0,00004$ & & & \\
\hline & & $\mathrm{c}=0,000000$ & & & \\
\hline
\end{tabular}

De acordo com a Tabela 2, os valores de $\mathrm{R}^{2}$ variaram entre 0,9731 e 0,9992. Os valores de EQM e $\chi^{2}$ variaram de 0,006 a 0,051 e de 0,00042 a 0,00291 , respectivamente. A equação que melhor se ajusta aos dados experimentais deve apresentar altos valores de $\mathrm{R}^{2} \mathrm{e}$ baixos valores de EQM, $\chi^{2}$ e P (Kaushal e Sharma, 2016). Segundo os autores anteriormente mencionados, valores de $\mathrm{P}$ inferiores a 5, indicam um excelente ajuste, enquanto valores maiores que 10 são indicativos de baixo ajuste. Dentre as equações utilizadas, a que apresentou maior valor de $\mathrm{R}^{2}$ e menores valores de EQM e $\chi^{2}$ para todos os tratamentos foi a equação de Wang \& Singh . Essa equação também apresentou bons ajustes na secagem de crambe (Costa, 2011). Segundo a Tabela 2, dentre as equações avaliadas, a equação de Wang \& Singh apresentou menores valores de $\mathrm{R}^{2}$. Durante a secagem de maracujá amarelo (Menezes, 2013), essa equação também apresentou baixos valores de $\mathrm{R}^{2}$. O caráter polinomial desta equação aliado à constante unitária para tempo igual a zero contribuem para o bom ajuste da mesma.

\section{Conclusão}

Foi possivel ajustar os dados experimentais de secagem convectiva de frutos de physalis, com e sem pré-tratamento físicos por congelamento a seis equações empiricas. Dentre as avaliadas, a que apresentou maior ajuste aos dados experimentais foi a de Wang \& Singh, com altos valores de $\mathrm{R}^{2}$ e baixos valores de EQM, $\mathrm{X}^{2}$ e $\mathrm{P}(\%)$.

\section{Agradecimentos}


Os autores agradecem a Fapemig, à CAPES e ao CNPq.

\section{REFERÊNCIAS:}

AOAC. Official Methods of Analysis, $18^{\text {th }}$ ed. Gaithersburg, MD: AOAC ,2010.

CORRÊA, J. L. G.; DEV, S. R. S.; GARIEPY, Y.; RAGHAVAN, G. S. V. Drying of Pineapple by Microwave-Vacuum with Osmotic Pretreatment. Drying Technology.,v. 29, p. 1556-1561, 2011.

COSTA, L. M.; RESENDE, O.; SOUSA, K. A.; GONÇALVES, D. N. Coeficiente de difusão efetivo e modelagem matematica da secagem de sementes de crambe. Revista Brasileira de Engenharia Agricola e Ambiental, v. 15, p. 1089, 2011.

CRANK, J. The mathematics of diffusion, $2^{\text {nd }} \mathrm{ed}, 1975$.

DERMESONLOUOGLOU, E. K.; POURGOURI, S.; TAOUKIS, P. S. Kinetic study of the effect of the osmotic dehydration pre-treatment to the shelf life of frozen cucumber. Innovative Food Science \& Emerging Technologies, v. 9, p. 542-549, 2008.

DOYMAZ, I.; ÖZDEMIR, Ö. Effect of air temperature, slice thickness and pretreatment on drying and rehydration of tomato. Int J Food Sci Technol., v. 49, p. 558-564, 2014.

DOYMAZ, İ. Hot-Air Drying and Rehydration Characteristics of Red Kidney Bean Seeds. Chem Eng Commun., v. 203, p. 599-608, 2016.

JUNQUEIRA, J. R. J.; MENDONÇA, K. S; CORRÊA, J. L. G. Microwave drying of sweet potato slices: influence of the osmotic pretreatment. Journal Defect and Diffusion Forum.,v. 367, p. 167-174, 2016.

JUNQUEIRA, J. R. J.; CORRÊA, J. L. G.; OLIVEIRA, H. M.; AVELAR, R. I. S.; PIO, L. A. S. Convective drying of cape gooseberry fruits: Effect of pretreatments on kinetics and quality parameters. Food Science and Technology, v. 82, p. 404-410, 2017.

KAUSHAL, P.; SHARMA, H. K. Osmo-convective dehydration kinetics of jackfruit (Artocarpus heterophyllus). J Saudi Soc Agric Sci .,v.15, p. 118-126, 2016.

KETATA, M.; DESJARDINS, Y.; RATTI, C. Effect of liquid nitrogen pretreatments on osmotic dehydration of blueberries. Journal Food Eng., v. 116, p. 202-212, 2013.

MENEZEZ, M. L.; SHOHER, A. P.; PEREIRA, N. C.; BARROS, S. T. D. Análise da cinética e ajustes de modelos matemáticos aos dados de secagem do bagaço de maracujá amarelo. Engevista., v. 15, p. 176-186, 2013.

RAMADAN, M. F. Bioactive phytochemicals, nutritional value, and functional properties of cape gooseberry (Physalis peruviana): An overview. Food Res Int., v. 44, p. 18301836, 2011.

SILVA, M. A. ; CORRÊA, J. L. G. . Academic Research on Drying in Brazil. Drying Technology, v. 23, p. 1345-1359, 2005.

ZIELINKA, M.; MICHALSKA, A. Microwave-assisted drying of blueberry (Vaccinium corymbosum L.) fruits: Drying kinetics, polyphenols, anthocyanins, antioxidant capacity, colour and texture. Food Chemistry., v. 212, p. 671-680, 2016. 